\title{
Garra dos líderes e capital psicológico dos liderados: Uma exploração concetual de condições moderadoras
}

\author{
Arménio Rego ${ }^{1}$, Filipa Sobral ${ }^{2}$, Ana Simões ${ }^{3}$, Cátia Duarte ${ }^{3}$, Francisco Reis ${ }^{3}$, Inês \\ Quaresma $^{3}$, Maria Guimarães ${ }^{3}$, Maria Teixeira ${ }^{3} \&$ Miguel Pina e Cunha $^{4}$ \\ ${ }^{1}$ Católica Porto Business School, Portugal \\ ${ }^{2}$ Universidade Católica Portuguesa, Porto, CEDH, Faculdade de Educação e Psicologia, Portugal \\ ${ }^{3}$ Universidade Católica Portuguesa, Centro Regional do Porto, Portugal \\ ${ }^{4}$ Nova School of Business and Economics, Portugal
}

\begin{abstract}
Resumo: A garra (termo aqui traduzido de grit) tem sido encarada como crucial para o sucesso dos indivíduos e a eficácia dos líderes, embora alguns autores não sustentem essa tese. A investigação empírica é escassa e pouco conclusiva. Um possível caminho para melhor compreender este construto passa por considerar condições moderadoras. Aqui discutimos três dessas condições: (1) garra veiculada pelo líder versus garra autoatribuída; (2) humildade expressa pelo líder; (3) apoio social expresso pelo líder. 0 modelo concetual argumenta que o líder que veicula mais garra perante os liderados desenvolve o capital psicológico destes, mas esse efeito é mitigado, ou mesmo anulado, se o líder for desprovido de humildade e não facultar apoio social aos liderados. Distintamente, o líder estimula o capital psicológico dos liderados se, além de veicular garra, também expressar humildade e apoio social aos liderados.
\end{abstract}

Palavras-chave: Liderança; garra (grit); garra veiculada versus garra autoatribuída; humildade; apoio social; capital psicológico.

Leaders' grit and followers psychological capital: Exploring moderating conditions: While it has been argued that grit (i.e., passion and perseverance for long-term goals) is crucial to the success of individuals and the effectiveness of leaders, some authors do not espouse such a perspective. Empirical research is scarce and inconclusive. We argue that, to better understand this construct, studies should include moderating conditions. We discuss three conditions: (1) conveyed leader grit versus self-attributed grit; (2) humility expressed by the leader; (3) social support expressed by the leader. In accordance with our conceptual model, (a) a leader who conveys high level of grit toward employees develop their psychological capital, (b) although this effect is mitigated or even nullified if the leader is devoid of humility and does not provide social support to employees. Distinctly, the leader fosters employees' psychological capital if, in addition to convey a high level of grit, he also expresses humility and social support toward employees.

Keywords: Leadership; grit; conveyed leader grit leader versus self-attributed grit; humility; social support; psychological capital.

O capital psicológico (Luthans et al., 2015), um elemento central do "comportamento organizacional positivo" e um importante construto dos "estudos organizacionais positivos" (Cunha et al., 2020; Youssef \& Luthans, 2012), tem vindo a ser apresentado como uma fonte de vantagem competitiva das organizações (Luthans \& Yousseff, 2004). 0 argumento é corroborado por evidência empírica sugerindo que o capital psicológico explica diversas atitudes e comportamentos organizacionalmente relevantes, designadamente o desempenho (Avey et al., 2011; Ferraro et al., 2020; Luthans \& Youssef-Morgan, 2017; Peterson et al., 2011; Walumbwa et al., 2010). Além disso, de um ponto de vista estritamente psicológico, a investigação sugere que o capital psicológico está associado a níveis superiores de saúde e bem-estar (Youssef-Morgan \& Luthans, 2015). A compreensão dos antecedentes do capital psicológico é, pois, um desafio importante, tanto ao nível teórico como no plano prático. A pesquisa sobre esses antecedentes é, todavia, modesta e empiricamente limitada (Luthans \& Youssef-Morgan, 2017), pelo que novos estudos são necessários. Neste artigo, consideramos que um líder pode exercer um papel importante no desenvolvimento do capital psicológico dos liderados se veicular um elevado nível de garra (Duckworth, 2016) para com os mesmos. Adicionalmente, sugerimos que esse papel é afetado por várias condições moderadoras, assim reforçando a necessidade de atenção à complexidade dos efeitos (condicionais) dos líderes sobre os liderados.

\footnotetext{
${ }^{1}$ Morada para correspondência: Arménio Rego, Rua de Diogo Botelho, 1327, 4169-005 Porto, Portugal. E-mail: arego@porto.ucp.pt
} 
A garra² ("paixão e perseverança em prol de objetivos de longo-prazo", Duckworth et al., 2007, p. 1087) é um traço não cognitivo, moderadoramente maleável e passível de desenvolvimento (Cunha et al., 2020; Duckworth, 2016; Rego et al., 2020). Tem sido considerada por diversos académicos como um ingrediente essencial para o sucesso e bem-estar psicológico do indivíduo em vários domínios (e.g., académico, laboral, desportivo e militar; Credé et al., 2017; Duckworth, 2016; Duckworth et al., 2007, 2009; Duckworth \& Gross, 2014; Mueller et al., 2017; von Cullin et al., 2014). Alguns "práticos" têm apontado no mesmo sentido. Por exemplo, Baer (2013) escreveu na revista Fast Company que, "para se ser bemsucedido, a garra é o ativo mais valioso". O mesmo entusiasmo tem sido assumido noutras publicações de negócios a propósito da relevância da garra para a eficácia dos líderes. Por exemplo, Mochari (2014) escreveu que "os verdadeiros líderes têm verdadeira garra". Stoltz (2015, p. 49) argumentou: "[A garra] é o motor da realização humana. É algo que todo o líder tem e, como a pesquisa revela, é mais valioso do qualquer outro traço nas pessoas quando tentam alcançar algo notável. Como líder, você sabe que a GARRA é o que separa as pessoas que se lamentam daquelas que cruzam a linha da meta. Distingue os melhores do resto, e por isso é tão desejável".

Alguns académicos são, porém, mais céticos. Credé et al. (2017) e Ivcevic e Bracket (2014) distanciaram-se do conceito devido aos resultados inconclusivos. Uma crítica foca-se no alegado fraco valor preditivo da garra para o sucesso e o desempenho das pessoas. Outra crítica, porventura mais enfática, é a de que a garra apresenta grande redundância com o construto da conscienciosidade, pelo que, alegadamente, não acrescenta conhecimento ao já existente sobre essa dimensão da personalidade. Acresce que a evidência acerca da relação entre a garra dos líderes e a sua eficácia é escassa. Uma meta-análise recente (Credé et al., 2017) menciona apenas um trabalho (uma dissertação de doutoramento; Davidson, 2014) que estuda como a garra e a esperança autoatribuídas dos líderes explicam a liderança transformacional também autoatribuída. Gilson et al. (2017) verificaram, em contexto militar, que a garra não explica a eficácia da liderança dos pelotões. Este resultado é surpreendente se atendermos à dureza do contexto militar. Mas é menos surpreendente se considerarmos que os líderes de pelotão operam em condições altamente estruturadas e não dispõem de grande autonomia (House \& Howell, 1992) que lhes permitiria traduzir a garra em desempenho mais elevado.

Ion et al. (2017), mesmo reconhecendo ser parca a atenção concedida pelos estudos à relevância da garra no trabalho, sugeriram que a garra "é dispensável na explanação das relações entre, por um lado, a personalidade e, por outro, a satisfação no trabalho" (p. 167). Essa perspetiva pessimista poderá ser manifestamente exagerada. Em primeiro lugar, a literatura sugere que, embora partilhem aspetos comuns, a garra e a conscienciosidade são distintos atributos individuais (Duckworth et al., 2007; Jordan et al., 2019; Rego et al., 2020; Schmidt et al., 2018). Por exemplo, a componente consistência de interesses (ou paixão) presente na garra está apenas modestamente representada na conscienciosidade. Ademais, os aspetos da confiabilidade e da inibição inerentes à conscienciosidade estão fracamente presentes na garra. 0 trabalho empírico de Rego et al. (2020) sustenta essa diferenciação e mostra que a garra, após controlado o efeito da conscienciosidade do líder, apresenta poder preditivo singular para a capacidade de os líderes influenciarem forças psicológicas dos liderados.

Em segundo lugar, é possível que a inconsistência da evidência empírica se deva à desconsideração de efeitos moderadores, isto é, de condições que reforçam, mitigam ou anulam os efeitos da garra em contextos de trabalho. Inspirando-nos em Credé et al. (2017), que sugeriram que o estudo da garra pode progredir se se tomarem em atenção as condições moderadoras, consideramos que a humildade dos líderes e o apoio social (veja Rego et al., 2020) que estes concedem aos liderados podem representar duas dessas condições.

O impacto de líderes como Mandela (Cascio \& Luthans, 2014) e Lincoln (Goodwin, 2005) assenta em qualidades como a garra. Mas a sua forte determinação (Collins, 2001) foi apoiada em qualidades como o conhecimento, a prudência e a humildade. Mesmo Steve Jobs, narcisista e determinado, desenvolveu a humildade que lhe permitiu transformar a Apple na empresa bem-sucedida que hoje se conhece (Owens et al., 2015). A garra foi uma condição necessária, mas não suficiente, para o sucesso desses líderes. Os méritos da garra dependem de como ela interage com outras qualidades positivas. Eis três exemplos (1) um líder pode ser determinado e arrogante, tornando-se incapaz de escutar outras pessoas e obter delas aconselhamento, sendo mais propenso a usar a sua garra para enveredar por objetivos irrealistas ou inviáveis; (2) um líder pode ser determinado e destrutivo, canalizando a sua garra para prosseguir objetivos perversos para as forças psicológicas dos liderados (Kellerman, 2004); (3) líderes incompetentes mas cheios de garra podem contaminar toda a organização com as suas decisões incompetentes, porventura reincidentes. Por conseguinte, os investigadores devem ser cautos a propósito dos alegados

2 “Garra" é um termo português (o outro é "fibra") que, na linguagem popular, reflete aquilo que na literatura anglo-saxónica é definido por Grit. Algumas traduções sugerem "força de caráter", mas esta expressão parece-nos desadequada porque incorpora teor moral que o conceito de grit não contempla. Neste artigo usamos o termo "garra", embora admitamos que possa não receber acolhimento entre outros académicos. 
méritos da garra dos líderes, e devem estudá-la no quadro de condições moderadoras. Neste trabalho concetual, advogamos que o papel dos líderes na promoção do capital psicológico dos liderados é condicionado por três condições moderadoras (Figura 1).

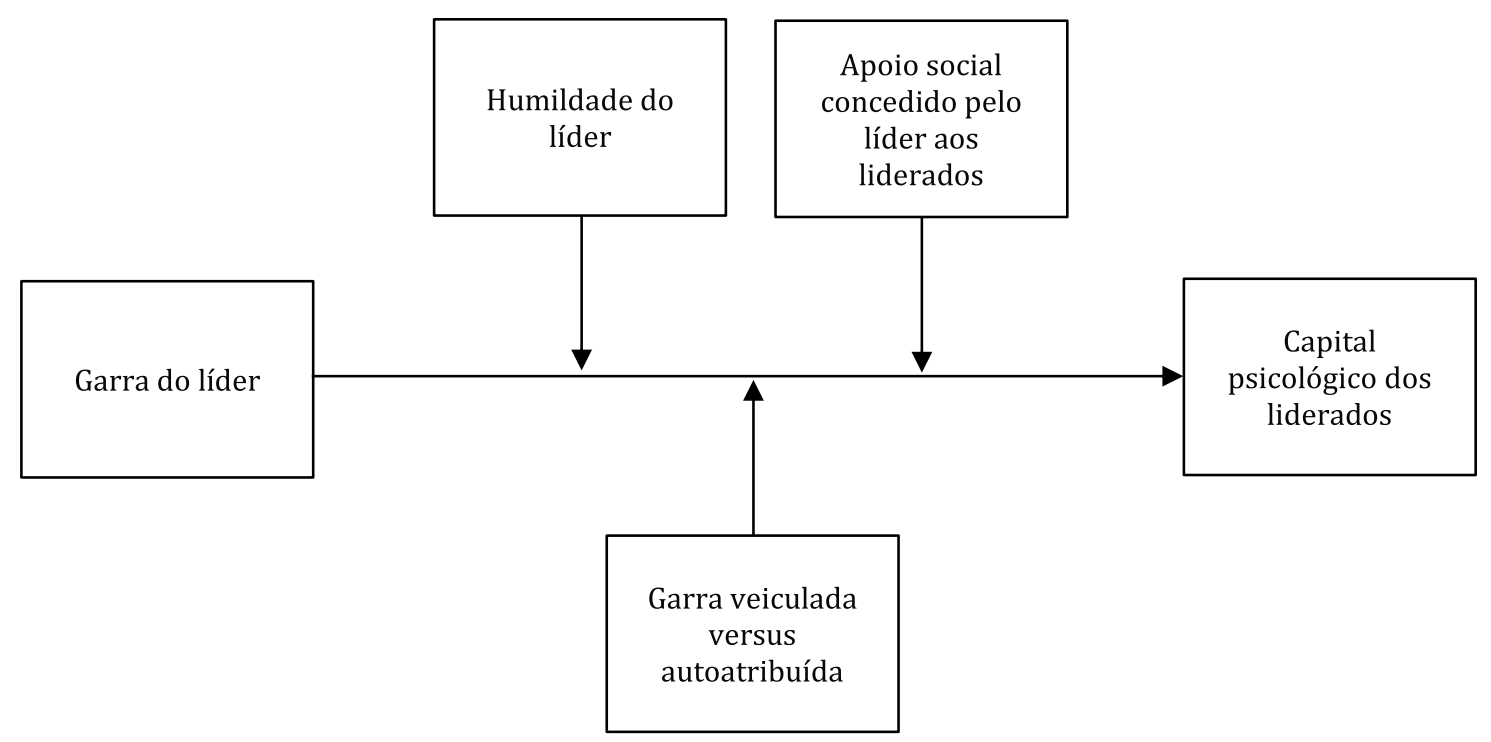

Figura 1. Modelo conceptual

O capital psicológico é um conjunto de quatro forças psicológicas mobilizadoras e motivadoras (autoeficácia, esperança, otimismo e resiliência) que tornam as pessoas mais eficazes no desempenho das suas funções e na prossecução de objetivos individuais e coletivos (Luthans et al., 2015). Consideramos que, através de processos de aprendizagem social e dos mecanismos emocionais e inferenciais sugeridos pela abordagem EASI ("emotions as social information"; Van Kleef, 2009), a determinação dos líderes com garra estimula o capital psicológico dos liderados (Luthans et al., 2015). Mas também sugerimos que este efeito é sujeito a três condições moderadoras. Antes de discuti-las, importa reconhecer que outras condições podem operar (e.g., capacidade de perdão dos líderes; traços de personalidade dos próprios liderados; segurança psicológica da equipa). No entanto, entendemos que as três condições aqui discutidas são pertinentes, e que o seu estudo pode abrir caminho à consideração de outras.

A primeira condição aqui discutida é de natureza metodológica: importa distinguir concetualmente a garra autoatribuída (e medida pelo autorrelato) e a garra veiculada (i.e., percecionada pelos liderados). Dada a natureza relacional da liderança (Uhl-Bien, 2006), a garra veiculada é porventura mais relevante do que a garra que o líder se atribui a si próprio (Rego et al., 2017a). Embora as duas variáveis possam estar relacionadas (i.e., veicula mais garra quem mais garra tem), é possível ser dotado de muita garra e não o demonstrar, assim como demonstrar uma garra que, interiormente, não se detém.

A segunda condição moderadora é a humildade (o sentimento de que não se é o "centro do universo"; Ou et al., 2014, p. 37). Um líder cheio de garra que não é humilde pode tornar-se impetuoso, incapaz de escutar os outros e de reconhecer erros e fragilidades, e obstinado na prossecução de objetivos inviáveis. Pode criar pressão desmedida sobre os liderados e encorajá-los a enveredar por ações que conduzem ao fracasso, por exemplo formulando objetivos excessivamente exigentes (Cunha et al., 2017; Rego et al., 2018). Foi precisamente isso que ocorreu na Volkswagen. A liderança da empresa, movida por objetivos impossiveis no que concerne à conjugação de baixos consumos de diesel com baixas emissões poluentes, gerou pressões sobre os liderados que os levaram a cometer a fraude que tanto escândalo provocou (Ewing, 2017). Neste artigo argumentamos que pressões deste teor podem exercer efeitos perversos sobre o desenvolvimento do capital psicológico dos liderados. Como discutiremos abaixo, consideramos que a garra veiculada pelo líder promove o capital psicológico dos liderados apenas se o líder também for humilde. Um líder dotado de garra, mas pouco humilde, pode tornar-se disfuncional (MacNamara et al., 2014) e dificultar o desenvolvimento do capital psicológico dos liderados.

A terceira condição é a perceção, pelos liderados, do apoio social do líder. Perceções positivas reforçam os processos de contágio e aprendizagem social que convertem a garra do líder em capital psicológico dos liderados. Essas perceções positivas também asseguram ao liderado que disporá de recursos (informacionais, sociais e emocionais) que lhe permitem enveredar por objetivos desafiantes, restaurar a resiliência em momentos críticos e de fracasso, e encarar o futuro com otimismo. Distintamente, 
perceções de baixo apoio social podem mitigar, ou mesmo neutralizar, esses efeitos encorajadores (Feeney \& Collins, 2015; Wu \& Parker, 2017). Em suma, os recursos emergentes do apoio social permitem ao liderado prosseguir as metas desafiantes e ambiciosas que um líder dotado de garra pretende alcançar. Se o líder não for apoiante, os liderados podem sentir-se desprotegidos perante os riscos associados a essas metas.

Para explorar concetualmente este tema, o artigo está organizado do seguinte modo: Começamos por distinguir a garra veiculada da garra autoatribuída, e mostramos porque a primeira é mais relevante para a promoção do capital psicológico dos liderados. Discutimos, depois, o efeito moderador da humildade e do apoio social percecionado. Exploramos, ainda, outros potenciais efeitos moderadores. No final, apresentamos algumas conclusões. 0 artigo faculta pistas concetuais que podem abrir caminho ao teste empírico das proposições sugeridas, assim como suscitar a exploração de novas variáveis moderadoras em estudos futuros. 0 que aqui propomos ajuda também a compreender porque o debate sobre os méritos (ou deméritos) da garra como construto pertinente pode beneficiar da consideração de boundary conditions em estudos futuros.

\section{Garra autoatribuída versus garra veiculada}

A maioria dos investigadores tem medido a garra através de autorrelatos e considerado que a garra autoatribuída e a garra descrita por observadores são medidas válidas do mesmo construto ("Os nossos resultados sugerem que a garra pode ser avaliada, de modo fiável, por informantes"; Duckworth \& Quinn, 2009, p. 170). Neste artigo, adotamos uma perspetiva distinta: a garra autoatribuída e a garra percecionada pelos liderados (aqui denominada "garra veiculada") são concetualmente distintas. Pelo menos três razões podem explicar essa diferença.

Em primeiro lugar, os líderes adotam frequentemente táticas de gestão de impressões para influenciar os seus interlocutores (Bolino et al., 2016), incluindo os liderados. Por exemplo, para inspirarem a equipa, podem expressar paixão por um objetivo de longo-prazo que, de facto, não perfilham. Em situações de crise ou dificuldades económico-financeiras, e perante obstáculos e riscos de fracasso, os líderes podem esconder dúvidas e hesitações, de modo a manterem os liderados focados e empenhados (Humphrey, 2012). Noutros momentos, podem esconder a paixão perseverante por um objetivo de longoprazo, por temerem que as mudanças necessárias para prosseguir esse objetivo suscitem resistências.

Em segundo lugar, os líderes operam no seio de "situações fortes" (Dalal et al., 2015). Estas situações podem obrigar o líder a adotar condutas que se compaginam mais com as exigências da situação do que com a sua própria personalidade (Dalal et al., 2015; Ng et al., 2008). Nessas situações, o líder pode sentir necessidade de veicular um nível de garra que realmente não possui. Logo, o seu comportamento ficará refletido na garra veiculada, não necessariamente na garra autoatribuída.

Em terceiro lugar, de acordo com a abordagem da liderança diferenciada (Wu et al., 2010; Zhang et al, 2015), um líder pode expressar diferentes níveis de garra perante diferentes liderados. E mesmo que um líder expresse realmente o mesmo nível perante todos os liderados, a garra veiculada será diferente: sendo a liderança um processo socialmente construído, os liderados desenvolvem diferentes perceções e interpretações acerca das mesmas condutas do líder (Van Kleef et al., 2009).

Por conseguinte, a garra autoatribuída e a garra veiculada devem ser consideradas concetualmente distintas. Enquanto a autoatribuída pode ser considerada um traço da personalidade interiormente experienciada (ou uma faceta da identidade individual; Oh et al., 2011), a garra veiculada representa as manifestações comportamentais de garra que não correspondem necessariamente à garra autoatribuída (Rego et al., 2017a). Considerando a natureza relacional da liderança (Uhl-Bien, 2006), a garra veiculada é mais relevante do que a autoatribuída para as reações dos liderados. Quando interagem com os liderados, os líderes veiculam, conscientemente ou não, os seus estados afetivos e as suas cognições, de modo verbal e não-verbal (Visser et al., 2013). A garra veiculada manifesta-se através de demonstrações de confiança, perseverança e energia dirigida à prossecução de objetivos (Hill et al., 2016; Lucas et al., 2015; Vainio \& Daukantaite, 2016). Estas manifestações comportamentais refletem apenas parcialmente o que o líder realmente pensa, sente, acredita e experiencia internamente (i.e., a garra autoatribuída). Considerando a teoria da aprendizagem social (Bandura, 1977) e os efeitos de contágio social (Chartrand \& Lakin, 2013), e abordando a liderança como processo de influência social, a garra veiculada releva mais do que a garra autoatribuída.

\section{A garra dos líderes e o capital psicológico dos liderados}

O capital psicológico é um estado positivo de desenvolvimento caraterizado por quatro componentes: autoeficácia, esperança, otimismo e resiliência (Luthans et al., 2015). A autoeficácia (ou autoconfiança) representa a confiança do indivíduo em que será capaz de envidar os necessários esforços para ser bemsucedido em tarefas desafiantes. 0 otimismo é um fenómeno atribucional. Um indivíduo otimista atribui o 
sucesso a si próprio e acredita que o mesmo se repetirá no tempo e noutras circunstâncias; e atribui o fracasso a fatores alheios, considerando que o mesmo não se repetirá noutros momentos nem em distintas circunstâncias. A esperança representa a conjugação de força de vontade (willpower) com capacidade para encontrar caminhos alternativos (waypower), quando outros se revelam inviáveis, para alcançar objetivos. A resiliência é a capacidade de perseverar, crescer e ser bem-sucedido perante obstáculos, erros e fracassos. As sinergias entre estas quatro forças sugerem que o capital psicológico seja considerado um construto nuclear, ou um fator de segunda-ordem constituído pelas quatro componentes (Luthans \& Youssef-Morgan, 2017). Sendo um estado psicológico, o capital psicológico é passível de desenvolvimento. Consideramos que a garra veiculada pelos líderes aos liderados pode estimular o capital psicológico destes, através dos processos de aprendizagem social e dos caminhos emocionais e inferenciais sugeridos pela abordagem EASI (Van Kleff, 2009).

A teoria da aprendizagem social sugere que as crenças, atitudes e ações dos liderados são aprendidas a partir do ambiente em que operam, através da observação e da aprendizagem vicariante. Por conseguinte, a garra veiculada pelos líderes encoraja o capital psicológico dos liderados através da modelação de papéis: o líder é um modelo com o qual os liderados aprendem. Como sugeriram Duckworth et al. (2007), a garra pode assentar em crenças de autoeficácia. Estas crenças são "captadas" pelos liderados que, através da modelação de papéis, desenvolvem a sua própria autoeficácia (Luthans et al., 2015; Luthans \& YousefMorgan, 2017). Indivíduos que se sentem liderados por um líder que persevera apesar dos fracassos, dificuldades e desaires, também aprendem a desenvolver esperança - ou seja, maior determinação para prosseguir objetivos, bem como maior capacidade para encontrar caminhos alternativos e prossegui-los quando outras vias e planos se mostram inviáveis (Luthans \& Youssef-Morgan, 2017). Os líderes que veiculam maior garra expressam otimismo quando fracassam ou falham. Através da modelação de papéis, os liderados também desenvolvem o seu otimismo. Os liderados também adquirem maior resiliência porque os líderes que veiculam maior garra mostram ser capazes de perseverar perante desaires, fracassos e adversidades (Kleiman et al., 2013; Duckworth, 2016).

A abordagem EASI (Van Kleef, 2009) sugere ainda que a garra veiculada pelos líderes estimula o capital psicológico dos liderados. À luz dessa abordagem, as expressões emocionais dos líderes facultam informação aos liderados e estes reagem através de dois caminhos - um inferencial-cognitivo e outro emocional - que os conduzem a mudanças comportamentais. Atendendo a que a garra veiculada se manifesta, e é interpretada, através de demonstrações de confiança, determinação, resiliência e energia (Hill et al., 2016; Lucas et al., 2015; Vainio \& Daukantaite, 2016), os liderados usam essa informação para avaliar positivamente a situação e, nas múltiplas interações com o líder, acabam por desenvolver o seu próprio capital psicológico (Van Kleef et al., 2010; Van Knippenberg \& Van Kleef, 2016).

Os dois caminhos sugeridos pela abordagem EASI operam neste processo. Por um lado, as emoções positivas, a energia e as expectativas associadas a elevados níveis de garra veiculada são transferidas para os liderados mediante processos de contágio emocional (Sy \& Choi, 2013; Visser et al., 2013); e este efeito emocional positivo facilita a construção e a restauração de recursos psicológicos, designadamente o capital psicológico (Fredrickson, 2001; Luthans \& Youssef-Morgan, 2017; Tugade \& Fredrickson, 2004). Por outro lado, as expressões emocionais associadas à garra veiculada também acionam os processos inferenciais (i.e., sentimentos, motivos, objetivos e intenções comportamentais) sugeridos pela abordagem EASI (Van Kleef, 2009; Van Kleef et al., 2010). Observando a forte garra veiculada pelo líder e as correspondentes emoções, expectativas e demostrações otimistas, os liderados podem inferir que o líder é autoconfiante, determinado (i.e., esperançoso), otimista e resiliente (Kleiman et al., 2013). Assim, quando veiculam forte garra, os líderes atuam como capacitadores do capital psicológico dos liderados. Daí as nossas proposições:

Proposição 1a. A garra veiculada pelos líderes prediz o capital psicológico dos liderados.

Proposição 1b. Comparativamente com a garra autoatribuída, a garra veiculada pelos líderes é um preditor mais forte do capital psicológico dos liderados.

\section{A humildade como condição moderadora}

Tal como ocorre com outras caraterísticas da personalidade presumivelmente brilhantes (Judge et al., 2009), a garra contém um lado escuro que pode mitigar ou neutralizar os efeitos positivos antes mencionados. Os líderes que veiculam mais garra podem sentir-se tentados a prosseguir cursos de ação inviáveis (Lucas et al., 2015; MacNamara et al., 2014) e a insistir em escolhas antes malsucedidas, apenas porque já foi feito investimento nessas escolhas (Arkes \& Blumer, 1985). Uma tal conduta é especialmente problemática porque a maior parte das atividades de um líder é levada a cabo em contextos repletos de ambiguidade que requerem flexibilidade, disponibilidade para abandonar projetos e planos, humildade para reconhecer limitações e erros, e capacidade de escutar os outros e obter conselhos (Weick, 2001). Por conseguinte, a menos que o líder expresse humildade, a sua inclinação para veicular uma imagem de grande 
garra pode ter menor impacto positivo sobre os liderados. Estes acabarão por desenvolver menor capital psicológico ao verificarem que o seu líder, repleto de perseverança e determinação, é incapaz de interromper projetos inviáveis, está mais preocupado em mostrar garra do que em tomar boas decisões, e é arrogante ao ponto de atribuir os fracassos aos liderados.

A humildade representa o grau em que o indivíduo "tem os pés assentes na terra" (Ou et al., 2014) é capaz de compreender as suas forças e fraquezas, bem como as forças e as qualidades dos outros, e está disponível para aprender com os outros e com os seus próprios erros (Owens et al., 2013). Um líder humilde é aberto a novos paradigmas, está disponível para aprender e acolher novas ideias, é recetivo a conselhos dos outros, e reconhece e está disposto a corrigir as suas próprias limitações e erros (Owens et al., 2013). Se o líder veicular elevada garra e expressar humildade, os liderados desenvolverão maior capital psicológico porque sentirão que os apaixonantes objetivos de longo-prazo do líder, que este prossegue com tenacidade, são realistas e fazíveis, e que o líder está disposto a mudar, ajustar-se e adaptar-se perante a realidade, os fracassos e as dificuldades.

Neste sentido, quando o líder veicula garra e expressa humildade, os efeitos positivos da elevada garra veiculada pelo líder têm maior probabilidade de se materializar. Os liderados percecionam que o líder não só persevera em prol de objetivos de longo-prazo, como também está disposto a aprender com os outros e a melhorar a qualidade da tomada de decisão. Um tal líder mostra que está disponível para aprender, e assim aumentar as probabilidades de sucesso; está consciente das suas limitações e mais disposto a enveredar por ações de autodesenvolvimento; abandona projetos inviáveis em prol de outros com maior probabilidade de sucesso; e valoriza os contributos dos liderados envolvendo-os na tomada de decisão. Veiculando elevada garra e expressando humildade, esse líder estimula o capital psicológico dos liderados: (a) aumenta-lhes a autoconfiança porque eles se sentem mais valorizados e dispõem de mais oportunidades para realizar tarefas em que são bem-sucedidos; (b) incrementa-lhes o otimismo porque eles ficam mais otimistas acerca do futuro ao lidarem com alguém que é flexível e segue caminhos viáveis - e não obstinado e cego à realidade; (c) estimula-lhes a esperança porque, ao sentirem que o líder é flexível e está recetivo a repensar processos, escolhas e planos, eles desenvolvem caminhos alternativos para lidar com problemas e oportunidades; e (d) aumenta-lhes a resiliência porque eles se sentem mais capazes de reduzir os riscos de resultados indesejáveis.

Por oposição, um líder que veicula elevada garra, mas não expressa humildade, mostra que não está disposto a atender à informação e conselhos dos outros, a abandonar perspetivas que contradizem as suas próprias crenças, ou a questionar a relevância e a pertinência dos seus objetivos. Consequentemente, os liderados não desenvolvem o seu capital psicológico (Luthans et al., 2015) e, como tal: (a) disporão de menores oportunidades para desenvolver a autoeficácia; (b) não desenvolverão otimismo acerca do futuro porque se sentirão incapazes de interromper cursos de ação iniciados pelo líder; (c) não incrementarão a sua esperança porque, antecipando resistências da parte do líder, se retrairão de sugerir caminhos alternativos para lidar com problemas e oportunidades - e evitarão repensar os objetivos (Luthans et al., 2015; Peterson et al., 2011); e (d) não se tornarão mais resilientes porque se sentirão incapazes de reduzir os riscos de efeitos perversos de más decisões.

Em casos extremos, um líder que expressa muita garra e pouca humildade pode mesmo reduzir o capital psicológico dos liderados. Ao tornar-se obstinado e arrogante, pode (a) impelir os liderados a prosseguir objetivos inviáveis, conduzindo-os a fracassos que reduzem a sua autoconfiança; (b) culpar os liderados por erros e fracassos, tornando-os menos otimistas, esperançosos e resilientes; (c) gerar-lhes sentimentos de impotência para interromper caminhos inviáveis, assim os tornando menos otimistas e esperançosos; (c) conduzi-los a menor resiliência por se sentirem incapazes de reduzir riscos de resultados indesejados provindos de persistentes más decisões. É mesmo possível que estes líderes desenvolvam excesso de confiança e soberba (hubris), assim gerando efeitos destrutivos (Park et al., 2018). Estes efeitos podem gerar declínio no capital psicológico dos liderados. Daqui decorre a segunda proposição:

Proposição 2. A relação entre a garra veiculada pelo líder e o capital psicológico dos liderados é moderada pelo nível de humildade que o líder expressa. A relação positiva (a) é mais forte quando o líder que veicula elevada garra também expressa elevada humildade e (b) é mais fraca ou mesmo negativa quando o líder não expressa humildade.

\section{0 apoio social percecionado como condição moderadora}

A relação entre a garra veiculada pelo líder e o capital psicológico dos liderados pode também ser condicionada pelas perceções de apoio social que os liderados sentem receber do seu líder. A relação é previsivelmente mais forte quando o apoio social percecionado é mais forte. A literatura sobre contágio social sugere que o contágio é maior quando a distância social entre o percetor e o modelo é menor (Radel et al., 2015), e as perceções de apoio social que o líder proporciona podem reduzir a distância social e 
psicológica (Trope \& Liberman, 2010). Quando sentem que o seu líder, além de veicular forte garra, também é apoiante, os liderados desenvolvem relações de maior qualidade com o líder e experienciam mais oportunidades de contacto social com o líder, o que aumenta a probabilidade de contágio e de aprendizagem social. Os efeitos positivos da garra veiculada pelo líder sobre o capital psicológico dos liderados podem, assim, ser reforçados.

0 apoio social percecionado pode também ser interpretado como uma fonte de recursos (materiais, emocionais, informacionais e relacionais) importantes para a prossecução dos objetivos de trabalho (Vainio \& Daukantaite, 2016). Ao percecionarem que o líder é exigente (devido à garra expressa), os liderados sentem-se desafiados a prosseguir objetivos ambiciosos; e ao sentirem que ele é apoiante, sentem que disporão dos recursos necessários e que poderão socorrer-se do apoio do líder para lidarem com riscos, adversidades, erros e fracassos. Estes sentimentos tornam os liderados mais confiantes em si próprios, mais otimistas acerca do futuro, mais propensos a explorar caminhos alternativos para lidar com problemas e oportunidades, e mais capazes de encarar os obstáculos e reveses como oportunidades de crescer.

Diferentemente, as perceções de escasso apoio social provindo do líder com garra podem conduzir os liderados a sentirem que o líder é insensível às suas necessidades (Duckworth, 2016) e, assim, a experienciarem um mais baixo sentido de autodeterminação. Deste modo, os liderados podem sentir que, sem o apoio do líder, a prossecução de objetivos é arriscada e as probabilidades de sucesso são limitadas. Daqui pode resultar menor autoconfiança, menor determinação e menor otimismo dos liderados. Sem apoio social, os liderados podem perder resiliência, pois as relações sociais positivas são uma fonte de resiliência para lidar com riscos, reveses e dificuldades associadas a metas ambiciosas e desafiantes expressas pelo líder dotado de garra (Feeney \& Collins, 2015; Luthans et al., 2015; Ozbay et al., 2008).

Em suma, o potencial efeito positivo de uma elevada garra veiculada pelo líder pode ser enfraquecido se o líder não for também apoiante. Em algumas circunstâncias de baixo apoio social, os liderados podem mesmo sentir que os comportamentos desafiantes e exigentes do líder, que expressa forte garra, podem ser interpretados mais como uma ameaça do que uma oportunidade (Niessen et al., 2017), com consequências negativas para o seu capital psicológico. Daqui extraímos a seguinte proposição:

Proposição 3. A relação entre a garra veiculada pelo líder e o capital psicológico dos liderados é moderada pelo nível de apoio social que os liderados sentem receber do líder. A relação positiva (a) é mais forte quando o líder que veicula elevada garra também expressa elevado apoio social e (b) é mais fraca ou mesmo negativa quando esse líder expressa baixo apoio social.

\section{DISCUSSÃO}

As condições moderadoras antes discutidas constituem exemplos ilustrativos de como a relevância da garra deve ser encarada com cautela. A perspetiva otimista que encara a garra como universalmente desejável deve ser complementada com a noção de que o seu papel depende da ocorrência de algumas condições. 0 que aqui discutimos é que a garra demonstrada pelos líderes pode ser uma condição necessária para a produção de efeitos positivos sobre os liderados - mas não uma condição suficiente. A perspetiva pessimista que subestima a relevância da garra (por considerar que os resultados da investigação são escassos e inconclusivos) deve prestar atenção a essas condições, em vez de subestimar a importância deste novo construto. Os líderes que demonstram garra apenas são uma fonte de desenvolvimento das forças psicológicas das pessoas que lideram se forem também dotados de outras qualidades e atributos. A humildade é uma dessas qualidades - e merece aqui um comentário particular. Sendo uma virtude, pode também ser interpretada, pelo menos em alguns contextos e junto de alguns tipos de liderados, como uma fraqueza do líder (Hu et al., 2018; Rego, 2019; Yuan et al., 2018). Mas o que este artigo indiretamente sugere é que esse risco é menor se o líder, além de humilde, for dotado de garra (Collins, 2001).

Além das condições moderadoras aqui discutidas, outras podem ser abrangidas pela investigação. Por exemplo, a relação entre a garra veiculada pelo líder e as reações dos liderados pode ser moderada por caraterísticas individuais destes (e.g., o seu nível de garra e a sua sensibilidade à garra do líder; Uhl-Bien et al., 2014). Outros atributos e comportamentos do líder podem também atuar como condições moderadoras. Por exemplo, é possível que a liderança servidora (Liden et al., 2014; van Dierendonck \& Patterson, 2015; van Dierendonck et al., 2014) reforce o papel positivo da garra veiculada pelo líder, e que o narcisismo, o maquiavelismo, a psicopatia do líder (Furtner et al., 2017) ou perfis de liderança destrutiva (Krasikova et al., 2013) e não ética (Brown \& Mitchell, 2010) atenuem esse efeito positivo ou reforcem o lado negro da garra. A garra autoatribuída pode também interagir com a garra veiculada. Serão os líderes mais impactantes os que veiculam maior garra e que são, simultaneamente, dotados de garra interior (i.e., autoatribuída) elevada? Será um baixo nível de garra autoatribuída um neutralizador da garra veiculada? 
O clima e as caraterísticas da equipa podem também atuar como condições moderadoras. Por exemplo, é possível que a garra veiculada pelo líder suscite efeitos mais positivos se a equipa for dotada de um clima de confiança e segurança psicológica (Edmondson \& Lei, 2014). Nessa condição, os liderados sentir-se-ão mais propensos a arriscar e experimentar novas soluções em resposta aos desafios e exigências do líder dotado de elevada garra. Dado que os líderes veiculam diferentes níveis de garra para diferentes liderados, importa compreender se o nível de (in)consistência da garra veiculada afeta o funcionamento da equipa. É possível, por exemplo, que os efeitos, positivos ou negativos, da garra veiculada pelo líder sejam reforçados (versus atenuados) quando todos os liderados percecionam que o líder é dotado do mesmo (versus diferente) nível de garra (Rego et al., 2017, 2019a, 2019b). Naturalmente, as próprias condições moderadoras podem interagir entre si. Um líder que veicula elevada garra e expressa humildade pode não ser apoiante. É possível que o efeito reforçador da humildade seja maior (versus menor) quando o líder é (versus não é) apoiante.

Outra limitação do artigo é que discute apenas o capital psicológico dos liderados como variável dependente. Importa que outras variáveis (como o desempenho) sejam contempladas nos estudos. A investigação ao nível das equipas deve também ser explorada. Um líder comunica com todos os membros da equipa e pode afetar as dinâmicas coletivas através do modo como expressa garra e denota humildade e outras caraterísticas pessoais.

Finalmente, alguns desafios metodológicos devem também ser considerados em pesquisas futuras, dos quais destacamos dois. Primeiro: o estudo de atributos (e.g., garra e humildade) veiculados recomenda que se considere diferenças nas "mensagens" veiculadas a diferentes membros das equipas (Wu et al., 2010; Zhang et al, 2015). Esta abordagem requer uma análise multinível ou, pelo menos, ao nível de equipa. Segundo: a maior parte dos estudos é de natureza correlacional, o que não permite suportar devidamente os nexos de causalidade. Estudos longitudinais e, idealmente, estudos experimentais são recomendáveis. No mínimo, para reduzir os riscos de variância do método comum, é conveniente recolher dados em fontes diferentes. As dificuldades operacionais aumentam, mas aumenta também a validade dos estudos.

\section{CONCLUSÃO}

A garra, como caraterística individual não cognitiva, tem sido alvo de controvérsia. Há quem subestime a sua relevância, é há quem a sobrevalorize. Consideramos que os investigadores poderão beneficiar com a inclusão nos seus estudos de condições moderadoras que reforçam ou atenuam os lados positivo e negativo dessa caraterística individual. Neste artigo, discutimos como essa investigação pode ser especialmente valiosa para compreender o impacto dos líderes nos liderados e nas suas equipas. Collins (2011) argumentou que "os executivos mais transformadores possuem uma combinação paradoxal de humildade pessoal e de força profissional. São tímidos e ferozes. Tímidos e destemidos” (p. 67) Consideramos que é recomendável investigar empiricamente o tema e explorar em que condições os líderes "destemidos" e "ferozes" são, ou não, construtores de melhores equipas e agentes desenvolvimentistas dos liderados. Também defendemos que, para compreender o impacto dos líderes nas suas equipas, é mais importante estudar a garra que eles veiculam do que a garra que se autoatribuem em escalas de auto-reporte.

\section{REFERÊNCIAS}

Arkes, H. R., \& Blumer, C. (1985). The psychology of sunk costs. Organizational Behavior and Human Decision Processes, 35, 124-140. https://doi.org/10.1016/0749-5978(85)90049-4

Avey, J. B., Reichard, R. J., Luthans, F., \& Mhatre, K. H. (2011). Meta-analysis of the impact of positive psychological capital on employee attitudes, behaviors, and performance. Human Resource Development Quarterly, 22(2), 127-152. https://doi.org/10.1002/hrdq.20070

Baer, D. (2013). To be successful, grit is a most valuable asset. Fast Company, October 1. http://www.fastcompany.com/3018841/leadership-now/to-be-successful-is-grit-moreimportant-than-intelligence

Bandura, A. (1977). Social learning theory. Prentice-Hall.

Bolino, M., Long, D., \& Turnley, W. (2016). Impression management in organizations: Critical questions, answers, and areas for future research. Annual Review of Organizational Psychology and Organizational Behavior, 3, 377-406. https://doi.org/10.1146/annurev-orgpsych-041015-062337

Brown, M. E., \& Mitchell, M. S. (2010). Ethical and unethical leadership: Exploring new avenues for future research. Business Ethics Quarterly, 20(4), 583-616. https://doi.org/10.5840/beq201020439

Cascio, W. F. \& Luthans, F. (2014). Reflections on the metamorphosis at Robben Island: The role of institutional work and positive psychological capital. Journal of Management Inquiry, 23(1), 51-67. https://doi.org/10.1177/1056492612474348 
Rego, Sobral, Simões, Duarte, Reis, Quaresma, Guimarães, Teixeira \& Pina e Cunha

Chartrand, T. L. \& Lakin, J. L. (2013). The antecedents and consequences of human behavioral mimicry. Annual Review of Psychology, 64, 285-308. https://doi.org/10.1146/annurev-psych-113011143754

Collins, J. (2001). Level 5 leadership: the triumph of humility and fierce resolve. Harvard Business Review, January, 67-76. https://hbr.org/2001/01/level-5-leadership-the-triumph-of-humility-and-fierceresolve-2

Credé, M., Tynan, M. C., \& Harms, P. D. (2016). Much ado about grit: A meta-analytic synthesis of the grit literature. Journal of Personality and Social Psychology, 113(3), 492-511. https://doi.org/10.1037/pspp0000102

Cunha, M.P., Giustiniano, L., Rego, A. \& Clegg, S. (2017). Mission impossible? The paradoxes of stretch goal setting. Management Learning, 48(2), 140-157. https://doi.org/10.1177/1350507616664289

Cunha, M.P., Rego, A., Simpson, A.V., \& Clegg, S. (2020). Positive organizational behavior: A reflective approach. Routledge.

Dalal, R. S., Meyer, R. D., Bradshaw, R. P., Green, J. P., Kelly, E. D., \& Zhu, M. (2015). Personality strength and situational influences on behavior: A conceptual review and research agenda. Journal of Management, 41(1), 261-287. https://doi.org/10.1177/0149206314557524

Davidson, B.A. (2014). Examining the relationship between non-cognitive skills and leadership: The influence of hope and grit on transformational leadership behavior. Unpublished doctoral dissertation. University of Kansas, Lawrence, Kansas. http://hdl.handle.net/1808/18643

Duckworth, A. (2016). Grit: The power of passion and perseverance. Scribner.

Duckworth, A. L. \& Quinn, P. D. (2009). Development and validation of the short grit scale. Journal of Personality Assessment, 91(2), 166-174. https://doi.org/10.1080/00223890802634290

Duckworth, A. L., \& Gross, J. (2014). Self-control and grit: Related but separable determinants of success. Current Directions in Psychological Science, 23(5), 325. https://doi.org/10.1177/0963721414541462

Duckworth, A. L., Peterson, C., Matthews, M. D., \& Kelly, D. R. (2007). Grit: Perseverance and passion for long-term goals. Journal of Personality and Social Psychology, 92(6), 1087-1101. https://doi.org/10.1037/0022-3514.92.6.1087

Duckworth, A.L., Quinn, P.D., Seligman, M.E. (2009). Positive predictors of teacher effectiveness. Journal of Positive Psychology, 4, 540-547. https://doi.org/10.1080/17439760903157232

Edmondson, A. C. \& Lei, Z. (2014). Psychological safety: The history, renaissance, and future of an interpersonal construct. Annual Review of Organizational Psychology and Organizational Behavior, 1, 23-43. https://doi.org/10.1146/annurev-orgpsych-031413-091305

Ewing, J. (2017). Faster, higher, farther: The inside story of the Volkswagen scandal. Random House.

Feeney, B. C. \& Collins, N. L. (2015). A new look at social support: A theoretical perspective on thriving through relationships. Personality and Social Psychology Review, 19(2), 113-147. https://doi.org/10.1177/1088868314544222

Ferraro, T., Pais, L., Moreira, J. M., \& Dos Santos, N. R. (2018). Decent work and work motivation in knowledge workers: The mediating role of psychological capital. Applied Research in Quality of Life, 13(2), 501-523. https://doi.org/10.1007/s11482-017-9539-2

Fredrickson, B. L. (2001). The role of positive emotions in positive psychology: The broaden-and-build theory of positive emotions. American Psychologist, 56(3), 218-226. https://doi.org/10.1037/0003066X.56.3.218

Furtner, M. R., Maran, T., \& Rauthmann, J. F. (2017). Dark leadership: The role of leaders' dark triad personality traits. In M. G. Clark \& C. W. Gruber (Eds), Leader Development Deconstructed (pp. 7599). Springer.

Gilson, T. A., Diz, M. A., \& Lochbaum, M. (2017). "Drive on": The relationship between psychological variables and effective squad leadership. Military Psychology, 29, 58-67. https://doi.org/10.1037/mil0000136

Goodwin, D. K. (2005). Team of rivals: The political genius of Abraham Lincoln. Simon \& Schuster.

Hill, P. L., Burrow, A. L., \& Bronk, K. C. (2016). Persevering with positivity and purpose: An examination of purpose commitment and positive affect as predictors of grit. Journal of Happiness Studies, 17(1), 257-269. https://doi.org/10.1007/s10902-014-9593-5

House, R. J., \& Howell, J. M. 1992. Personality and charismatic leadership. The Leadership Quarterly, 3, 81108. https://doi.org/10.1016/1048-9843(92)90028-E

Hu, J., Erdogan, B., Jiang, K., Bauer, T. N., \& Liu, S. (2018). Leader humility and team creativity: The role of team information sharing, psychological safety, and power distance. Journal of Applied Psychology, 103(3), 313-323. https://doi.org/10.1037/apl0000277 
Humphrey, R. H. (2012). How do leaders use emotional labor? Journal of Organizational Behavior, 33(5), 740-744. https://doi.org/10.1002/job.1791

Ion, A., Mindu, A., \& Gorbanescu, A. (2017). Grit in the workplace: Hype or ripe? Personality and Individual Differences, 111, 163-168. https://doi.org/10.1016/j.paid.2017.02.012

Ivcevic, Z., \& Brackett, M. (2014). Predicting school success: Comparing conscientiousness, Grit, and emotion regulation ability. Journal of Research in Personality, 52, 29-36. https://doi.org/10.1016/j.jrp.2014.06.005

Jordan, S. L., Ferris, G. R., Hochwarter, W. A., \& Wright, T. A. (2019). Toward a work motivation conceptualization of grit in organizations. Group \& Organization Management, 44(2), 320-360. https://doi.org/10.1177/1059601119834093

Judge, T. A., Piccolo, R. F., \& Kosalka, T. (2009). The bright and dark sides of leader traits: A review and theoretical extension of the leader trait paradigm. The Leadership Quarterly, 20, 855-875. https://doi.org/10.1016/j.leaqua.2009.09.004

Kellerman, B. (2004). Bad leadership: What it is, how it happens, why it matters. Harvard Business Press.

Kleiman, E. M., Adams, L. M., Kashdan, T. B., \& Riskind, J. H. (2013). Gratitude and grit indirectly reduce risk of suicidal ideations by enhancing meaning in life, Evidence for a mediated moderation model. Journal of Research in Personality, 47, 539-546. https://doi.org/10.1016/j.jrp.2013.04.007

Krasikova, D. V., Green, S. G., \& LeBreton, J. M. (2013). Destructive leadership: A theoretical review, integration, and future research agenda. Journal of Management, 39(5), 1308-1338. https://doi.org/10.1177/0149206312471388

Liden, R. C., Panaccio, A., Meuser, J. D., Hu, J., \& Wayne, S. (2014). Servant leadership: Antecedents, processes, and outcomes. In D. V. Vay (Ed.), The Oxford handbook of leadership and organizations (357-379). Oxford University Press.

Lucas, G. M., Gratch, J., Cheng, L., \& Marsella, S. (2015). When the going gets tough: Grit predicts costly perseverance. Journal of Research in Personality, 59, 15-22. https://doi.org/10.1016/j.jrp.2015.08.004

Luthans, F. \& Youssef-Morgan, C. M. (2017). Psychological capital: An evidence-based positive approach. Annual Review of Organizational Psychology and Organizational Behavior, 4, 339-366. https://doi.org/10.1146/annurev-orgpsych-032516-113324

Luthans, F., \& Youssef, C. M. (2004). Human, social, and now positive psychological capital management: Investing in people for competitive advantage. Organizational Dynamics, 33(2), 143-160. https://doi.org/10.1016/j.orgdyn.2004.01.003

Luthans, F., Youssef, C. M., \& Avolio, B. J. (2015). Psychological capital and beyond. Oxford University Press.

MacNamara, B. N., Hambrick, D. Z., \& Oswald, F. L. (2014). Deliberate practice and performance in music, games, sports, education, and professions: A meta-analysis. Psychological Science, 25, 1608-1618. https://doi.org/10.1177/0956797614535810

Mochari, I. (2014). True leaders have true grit. Inc, February 3. http://www.inc.com/the-buildnetwork/true-grit.html

Mueller, B. A., Wolfe, M. T., \& Syed, I. (2017). Passion and grit: An exploration of the pathways leading to venture success. Journal of Business Venturing, 32(3), 260-279. https://doi.org/10.1016/j.jbusvent.2017.02.001

Ng, K.-Y., Ang, S., \& Chan, K.-Y. (2008). Personality and leader effectiveness: A model of leadership selfefficacy, job demands, and job autonomy. Journal of Applied Psychology, 93(4), 733-743. https://doi.org/10.1037/0021-9010.93.4.733

Niessen, C., Mäder, I., Stride, C., \& Jimmieson, N. L. (2017). Thriving when exhausted: The role of perceived transformational leadership. Journal of Vocational Behavior, 103, 41-51. https://doi.org/10.1016/j.jvb.2017.07.012

Oh, I.-S., Wang, G., \& Mount, M. K. (2011). Validity of observer ratings of FFM personality traits: A metaanalysis. Journal of Applied Psychology, 96(4), 762-773. https://doi.org/10.1037/a0021832

Ou, A. Y, Tsui, A. S., Kinicki, A. J., Wladman, D. A., Xiao, Z., \& Song, L. J. (2014). Humble Chief Executive Officers' connections to top management team integration and middle managers' responses. Administrative Science Quarterly, 59, 34-72. https://doi.org/10.1177/0001839213520131

Owens, B. P., Johnson, M. D., \& Mitchell, T. R. (2013). Expressed humility in organizations: Implications for performance, teams, and leadership. Organization Science, 24(5), 1517-1538. https://doi.org/10.1287/orsc.1120.0795

Owens, B. P., Wallace, A. S., \& Waldman, D. A. (2015). Leader narcissism and follower outcomes: The counterbalancing effect of leader humility. Journal of Applied Psychology, 100(4), 1203-1213. https://doi.org/10.1037/a0038698 
Ozbay, F., Fitterling, H., Charney, D., \& Southwick, S. (2008). Social support and resilience to stress across the life span: A neurobiologic framework. Current Psychiatry Reports, 10, 304-310. https://doi.org/10.1007/s11920-008-0049-7

Park, J. H., Kim, C., Chang, Y. K., Lee, D. H., \& Sung, Y. D. (2018). CEO hubris and firm performance: Exploring the moderating roles of CEO power and board vigilance. Journal of Business Ethics, 47, 919-933. https://doi.org/10.1108/JSMA-04-2019-0049

Peterson, S. J., Luthans, F., Avolio, B. J., Walumbwa, F. O., \& Zhang, Z. (2011). Psychological capital and employee performance: A latent growth modeling approach. Personnel Psychology, 64, 427-450. https://doi.org/10.1111/j.1744-6570.2011.01215.x

Radel, R., Fournier, M., de Bressy, V., \& d'Arripe-Longueville, F. (2015). You're too much for me: Contagion of motivation depends on perceiver-model distance. Motivation and Emotion, 39(3), 374-383. https://doi.org/10.1007/s11031-014-9451-0

Rego, A. (2019). Liderança: Humildade e soberba. Sílabo.

Rego, A., Cavazotte, F., Cunha, M. P., Valverde, C., Meyer, M., \& Giustiniano, L. (2020). Gritty leaders promoting employees' thriving at work. Journal of Management. https://doi.org/10.1177/0149206320904765

Rego, A., Owens, B., Leal, S., Melo, A., Cunha, M. P., Gonçalves, L., \& Ribeiro, L. (2017). How leader humility helps teams to be humbler, psychologically stronger, and more effective: A moderated mediation model. The Leadership Quarterly, 28, 639-658. https://doi.org/10.1016/j.leaqua.2017.02.002

Rego, A., Owens, B., Yam, K. C., Bluhm, D., Cunha, M. P., Silard, T., Gonçalves, L., Martins, M., Simpson, A. V., \& Liu, W. (2019b). Leader humility and team performance: Exploring the mechanisms of team psychological capital and task allocation effectiveness. Journal of Management, 45(3), 1099-1033. https://doi.org/10.1177/0149206316688941

Rego, A., Valverde, C., Oliveira, E., \& Cunha, M. P. (2018). Gestão por objetivos. Lisboa: Sílabo.

Rego, A., Yam, K. C., Owens, B., Story, J. S., P, Cunha, M. P., Bluhm, D., \& Lopes, M. P. (2019a). Conveyed leader PsyCap predicting leader effectiveness through positive energizing. Journal of Management, 45(4), 1689-1712. https://doi.org/10.1177/0149206317733510

Schmidt, F. T., Nagy, G., Fleckenstein, J., Möller, J., \& Retelsdorf, J. (2018). Same same, but different? Relations between facets of conscientiousness and grit. European Journal of Personality, 32(6), 705-720. https://doi.org/10.1002/per.2171

Stoltz, P. G. (2015). Leadership grit. Leader to Leader, 78, 49-55. https://doi.org/10.1002/ltl.20205

Sy, T. \& Choi, J.N. (2013). Contagious leaders and followers, exploring multi-stage mood contagion in a leader activation and member propagation LAMP model. Organizational Behavior and Human Decision Processes, 122, 127-140. https://doi.org/10.1016/j.obhdp.2013.06.003

Trope, Y. \& Liberman, N. (2010). Construal-level theory of psychological distance. Psychological Review, 117(2), 440-463. https://doi.org/10.1037/a0018963

Tugade, M. M. \& Fredrickson, B. L. (2004). Resilient individuals use positive emotions to bounce back from negative emotional experiences. Journal of Personality and Social Psychology, 86(2), 320-333. https://doi.org/10.1037/0022-3514.86.2.320

Uhl-Bien, M. (2006). Relational leadership theory: Exploring the social processes of leadership and $\begin{array}{lllll}\text { organizing. The } & \text { Leadership }\end{array}$ http://dx.doi.org/10.1016/j.leaqua.2006.10.007

Uhl-Bien, M., Riggio, R. E., Lowe, K. B., \& Carsten, M. K. (2014). Followership theory: A review and research agenda. The Leadership Quarterly, 25(1), 83-104. https://doi.org/10.1016/j.leaqua.2013.11.007

Vainio, M. M. \& Daukantaite, D. (2016). Grit and different aspects of well-being: Direct and indirect relationships via sense of coherence and authenticity. Journal of Happiness Studies, 17(5), 21192147. https://doi.org/10.1007/s10902-015-9688-7

Van Dierendonck, D. \& Patterson, K. (2015). Compassionate love as a cornerstone of servant leadership: An integration of previous theorizing and research. Journal of Business Ethics, 128, 119-131. https://doi.org/10.1007/s10551-014-2085-z

Van Dierendonck, D., Stamn, D., Boersma, P., de Windt, N., \& Alkema, J. (2014). Same difference? Exploring the differential mechanisms linking servant leadership and transformational leadership to follower outcomes. The Leadership Quarterly, 25, 544-562. https://doi.org/10.1016/J.LEAQUA.2013.11.014

Van Kleef, G. A. (2009). How emotions regulate social life: The emotions as social information EASI model. Current Directions in Psychological Science, 18, 184-188. https://doi.org/10.1111/j.14678721.2009.01633.x

Van Kleef, G. A., De Dreu, C. K. W., \& Manstead, A. S. R. (2010). An interpersonal approach to emotion in social decision making: The emotions as social information model. Advances in Experimental Social Psychology, 42, 45-96. https://doi.org/10.1016/S0065-2601(10)42002-X 
Van Kleef, G. A., Homan, A. C., Beersma, B., van Knippenberg, D., van Knippenberg, B., \& Damen, F. (2009). Searing sentiment or cold calculation? The effects of leader emotional displays on team performance depend on follower epistemic motivation. Academy of Management Journal, 52, 562580. https://doi.org/10.5465/amj.2009.41331253

Van Knippenberg, D. \& Van Kleef, G. A. (2016). Leadership and affect, Moving the hearts and minds of followers. The Academy of Management Annals, 10, 799-840. https://doi.org/10.1080/19416520.2016.1160515

Visser, V. A., van Knippenberg, D., van Kleef, \& Wisse, B. (2013). How leader displays of happiness and sadness influence follower performance: Emotional contagion and creative versus analytical performance. The Leadership Quarterly, 24,172-188. https://doi.org/10.1016/j.leaqua.2012.09.003

Von Culin, K. R., Tsukayama, E., \& Duckworth, A. L. (2014). Unpacking grit: Motivational correlates of perseverance and passion for long-term goals. The Journal of Positive Psychology, 9(4), 306-312. https://doi.org/10.1080/17439760.2014.898320

Walumbwa, F. O., Peterson, S. J., Avolio, B., \& Hartnell, C. A. (2010). An investigation of the relationships among leader and follower psychological capital, service climate, and job performance. Personnel Psychology, 63, 937-963. https://doi.org/10.1111/j.1744-6570.2010.01193.x

Weick, K. E. 2001. Leadership as the legitimation of doubt. In W. Bennis, G. Spreitzer, \& T. Cummings (Eds.), The future of leadership: Today's top leadership thinkers speak to tomorrow's leaders (pp. 91-102). Jossey-Bass.

Wu, C. H. \& Parker, S. K. (2017). The role of leader support in facilitating proactive work behavior. Journal of Management, 43(4), 1025-1049. https://doi.org/10.1177/0149206314544745

Wu, J. B., Tsui, A. S., \& Kinicki, A. J. (2010). Consequences of differentiated leadership in groups. Academy of Management Journal, 53(1), 90-106. https://doi.org/10.5465/AMJ.2010.48037079

Youssef-Morgan, C. M. \& Luthans, F. (2015). Psychological capital and well-being. Stress and Health, 31(3), 180-188. https://doi.org/10.1002/smi.2623

Yuan, L., Zhang, L., \& Tu, Y. (2018). When a leader is seen as too humble: A curvilinear mediation model linking leader humility to employee creative process engagement. Leadership \& Organization Development Journal, 39(4), 468-481. https://doi.org/10.3389/fpsyg.2019.00673

Zhang, X.-A., Li, N., Ullrich, J., \& van Dick, R. (2015). Getting everyone on board: The effect of differentiated transformational leadership by CEOs on top management team effectiveness and leader-rated firm performance. Journal of Management, 1898-1933. https://doi.org/10.1177/0149206312471387

$\begin{array}{lr}\text { Historial do artigo } \\ \text { Recebido } & 12 / 2018 \\ \text { Aceite } & 07 / 2020 \\ \text { Publicado } & 08 / 2020\end{array}$

